\title{
Strong Scatterers Integrator Based on ADT in Non-Gaussian Clutter
}

\author{
Gu Xinfeng1, *, Hao Xiaolin², Yang Ganlin ${ }^{1}$, Li Xinxing ${ }^{1}$ \\ ${ }^{1}$ China Satellite Maritime Tracking \& Control Department, Jiangyin,China \\ ${ }^{2}$ Yantai Electricityn and Economy Technical Institute, Yantai, China
}

\section{Email address:}

y6ckgxf@126.com (Gu Xinfeng)

*Corresponding author

\section{To cite this article:}

Gu Xinfeng, Hao Xiaolin, Yang Ganlin, Li Xinxing. Strong Scatterers Integrator Based on ADT in Non-Gaussian Clutter. Science Discovery. Vol. 4, No. 1, 2016, pp. 26-30. doi: 10.11648/j.sd.20160401.15

Received: March 4, 2016; Accepted: March 12, 2016; Published: April 16, 2016

Abstract: This paper addresses range-spread target detection in compound Gaussion clutter modeled as spherically invariant random vector clutter (SIRV). A Strong scatterers integrator based on Anderson-Darling test (ADT-SSI) is addressed for the problem that MSDD has detection loss when falsely estimating the number of scatterers. For ADT-SSI, adaptively estimate the number of target scatterers according to the observations, which can improve detection performance and robustness of detector effectively.

Keywords: Non-Gaussian Clutter, Range-Spread Rarget, Detection, Anderson- Darling Test

\section{复合高斯背景下基于ADT的强散射点积累检测器}

\author{
顾新锋 ${ }^{1, *}$, 郝晓琳 ${ }^{2}$, 杨甘霖 $^{1}$, 李新星 ${ }^{1}$ \\ ${ }^{1}$ 中国卫星海上测控部, 江阴, 中国 \\ ${ }^{2}$ 烟台电力经济技术研究所, 烟台, 中国 \\ 邮箱 \\ y6ckgxf@126. com（顾新锋）
}

中文摘要: 本文研究了SIRV建模的复合高斯杂波背景下的距离扩展目标检测问题。针对MSDD检测器在估计目标散射点 个数估计失配时存在一定检测损失的问题, 提出了一种基于AD检验的强散射点积累检测器 (ADT-SSI)。首先, 利用 AD 检验估计目标散射点个数, 再利用估计的强散射点进行积累与门限比较。仿真结果和实测数据验证表明, 与MSDD相比 ADT-SSI具有更好的检测性能和鲁棒性。

关键词: 非高斯杂波, 距离扩展目标, 检测, $\mathrm{AD}$ 检验

\section{1. 引言}

与低分辨率雷达不同，高分辨率雷达能够在空间上把 一个目标分解成许多散射点, 目标回波在雷达径向上的多 个强散射点分布在不同的距离单元中, 形成距离扩展目标
$[1,2]$ 。目标的离散散射中心常被称为多主散射中心MDS (multiple dominant scattering)。增加雷达分辨率并采 用适当的检测方法能极大地提高检测概率 [3], 但如果采 用传统点目标检测器, 由于部分目标能量泄漏到参考单元 中, 检测性能将大大下降, 甚至完全失效 [4]。文献 [5-7] 
研究了高斯杂波背景下距离扩展目标的检测方法, 但在高 分辨率情况下, 雷达会接收到类似于目标的尖峰, 此时高 斯模型不再适用, 这种含尖峰的杂波可用球不变随机向量 SIRV (spherically invariant random vector) 来建模 $[2,8]$ 。文献 $[9]$ 在SIRV杂波条件下, 提出了不依赖于散射 点密度的广义似然比检验检测器NSDD (non-scatterer density dependent) 和基于目标散射点密度先验信息的检 测器SDD (scatterer density dependent)。由于SDD是统 计平均意义下的最优检测, 未能充分利用散射点密度的先 验信息, 存在一定的检测损失。文献 $[10]$ 有效利用目标散 射点信息, 获得了修正的检测器MSDD (modified SDD), 并 证明了MSDD的恒虚警率 (CFAR, constant false alarm rate) 特性。虽然MSDD的检测性能要优于NSDD和SDD, 但估 计的目标散射点个数与实际值不一致时, MSDD存在一定的 检测损失。为此, 文本利用AD检验 (Anderson-Darling test) 估计目标强散射点个数, 再对强散射点进行积累检测, 这 样, 检测器能够根据实际观测值估计散射点个数, 提高了 检测性能, 增强了系统的鲁棒性。

\section{2. 问题描述}

假设数据从 $N$ 个阵元得到, 需解决跨过 $K$ 个距离单元 $\boldsymbol{Z}_{t}$, $t=1,2, \cdots, k$ 检测一个目标存在与否的问题。假设可能的目 标完全包含在这 $K$ 个距离单元中, 并且忽略距离走动的问 题。考虑杂波为主的干扰环境, 忽略内部噪声的影响。所 要解决的检测问题可表示为:

$$
\begin{aligned}
& \mathrm{H}_{0}: \boldsymbol{z}_{t}=\boldsymbol{c}_{t}, \quad t=1, \ldots, K \\
& \mathrm{H}_{1}: \boldsymbol{z}_{t}=\alpha_{t} \boldsymbol{p}+\boldsymbol{c}_{t}, t=1, \ldots, K
\end{aligned}
$$

式中, $p$ 表示已知的单位导向矢量, 即 $p^{\mathrm{H}} p=1$ （符号 “ $(・)^{\mathrm{H}}$ ” 表示共轭转置); 标量 $\alpha_{t}$ 是反映目标和信道影响 的未知确定性参数。当 $\boldsymbol{p}=\left(1, \mathrm{e}^{\mathrm{j} \phi}, \mathrm{e}^{\mathrm{j} 2 \phi}, \ldots, \mathrm{e}^{\mathrm{j}(N-1) \phi}\right)^{\mathrm{T}} / \sqrt{N}$ 时 (其中 $\boldsymbol{\phi}$ 为常数,

符号 “ $(\cdot)^{\mathrm{T}}$ ” 表示转置), 式 (1) 表示的目标模型与移动距 离扩展目标模型相似 [6], 在那里假设目标在 $\mathrm{N}$ 个脉冲的相 干处理时间内无距离走动。

杂波回波用SIRV分布来建模 $[8]$, 则杂波向量 $\boldsymbol{c}_{t}$ 可以表 示成

$$
\boldsymbol{c}_{t}=\sqrt{\tau_{t}} \cdot \boldsymbol{\eta}_{t}, t=1, \ldots, K
$$

式中, $\boldsymbol{\eta}_{t}=\left(\boldsymbol{\eta}_{t}(1), \boldsymbol{\eta}_{t}(2), \cdots, \boldsymbol{\eta}_{t}(K)\right)^{\mathrm{T}}, \boldsymbol{\eta}_{t}(n), n=1,2, \cdots, N$ 是独立同分布 (IID, independent and identically distributed) 的零均值方差为 1 的复圆高斯随机变量; 纹 理分量 $\tau_{t}$ 是服从分布 $f_{\tau}$ 的非负随机变量, 用来描述不同距 离单元间杂波功率水平的起伏。根据SIRV模型可知, $\boldsymbol{\eta}_{t}$ 和 $\tau_{t}$ 是相互独立的, 且 $\boldsymbol{\eta}_{t}$ 的归一化协方差矩阵 $\boldsymbol{\Sigma}$ 表示为

$$
\Sigma=E\left\{\boldsymbol{\eta}_{t} \boldsymbol{\eta}_{t}^{\mathrm{H}}\right\}, t=1, \ldots, K
$$

其中 $\boldsymbol{\Sigma}$ 为正定的Hermitian矩阵。

\section{ADT-SSI检测器}

对于占据 $K$ 个距离单元稀疏散射点目标的回波，可以 分为两部分, 一部分是存在目标强散射点的回波, 另一部 分是纯杂波回波。对于雷达目标检测, 通常认为较强的回 波存在目标散射点, 而较弱的回波为不含目标散射点。这 样可以对回波序列进行排序, 若目标散射点个数为 $h_{0}$, 则 利用序列中最大的 $h_{0}$ 个值进行积累, 再与阈值进行比较, 就形成文献 [10]提出的MSDD检测器。MSDD在已知目标散射 点个数时具有较好的检测性能, 而当目标散射点个数未知 时, 需要事先进行估计, 当估计值与真实值不匹配时, 存 在一定的检测性能损失, 特别地当估计值 $h_{e}<h_{0}$ 时, 由 于部分存在目标散射点的距离单元没有被积累, MSDD存在 较大的检测损失, 这一点将在后面的仿真实验中得到验证。 针对该问题, 本节给出一种基于 $\mathrm{AD}$ 检验的目标散射点个数 估计方法。

\section{1. ATD-SSI检测器}

令集合 $\Theta_{h_{0}}$ 表示存在目标散射点的距离单元下标的 集合, $h_{0}$ 表示目标强散射点所占距离单元个数, $h_{\mathrm{e}}$ 表示 $h_{0}$ 的估计值。在未知 $\alpha_{t}$ 和 $f_{\tau}$ 的条件下, 对SDD进行修正, 可得 MSDD的检测统计量为 [10]

$$
\lambda_{\mathrm{MSDD}}=-2(N-1) \sum_{t \in \Theta_{h_{0}}} \ln \left(1-w_{t}\right)
$$

其中,

$$
w_{t}=\frac{\left|\boldsymbol{p}^{\mathrm{H}} \boldsymbol{\Sigma}^{-1} \boldsymbol{z}_{t}\right|^{2}}{\left(\boldsymbol{z}_{t}^{\mathrm{H}} \boldsymbol{\Sigma}^{-1} \boldsymbol{z}_{t}\right)\left(\boldsymbol{p}^{\mathrm{H}} \boldsymbol{\Sigma}^{-1} \boldsymbol{p}\right)}, t=1, \ldots, K
$$

将 $W_{t}$ 按从小到大的顺序排列, 可得有序序列 $W_{(1)} \leqslant W_{(2)}$ $\leqslant \bullet ・-\leqslant W_{(k)}$ 。利用 $W_{(k)}, k=1,2, \cdots, K$ 中最大的 $h_{0}$ 个值进行 积累可得SSI检测统计量为:

$$
\lambda_{\mathrm{SSI}}=-2(N-1) \sum_{k=K-h_{0}+1}^{K} \ln \left(1-w_{(k)}\right)
$$

当 $h_{0}=K$ 时, $\mathrm{SSI}$ 退化为 $\mathrm{NSDD}[9]$

$$
\lambda_{\mathrm{NSDD}}=-2(N-1) \sum_{k=1}^{K} \ln \left(1-w_{(k)}\right)
$$

当目标散射点个数未知时, 可以利用 $A D$ 检验对强散射 点个数进行估计, 再对强散射点进行积累, 这时检测统计 量为

$$
\lambda_{\text {ADT-SSI }}\left(h_{\mathrm{e}}\right)=-2(N-1) \sum_{k=K-h_{\mathrm{e}}+1}^{K} \ln \left(1-w_{(k)}\right)
$$

因此, ATD检测器可以表示为 


$$
\lambda_{\text {ADT-SSI }}\left(h_{\mathrm{e}}\right) \underset{\underset{\mathrm{H}}{<}}{\stackrel{\mathrm{H}_{1}}{>}} T_{h_{\mathrm{e}}}
$$

式中, $T_{h_{\mathrm{e}}}$ 表示 $h_{\mathrm{e}}$ 和强散射点积累对应的检测门限。

由于ATD-SSI在本质上与MSDD具有一致性, 因此由文 献 $[10]$ 的分析可知, 与MSDD类似, ATD-SSI也具有CFAR特 性。比较式 (8) 和式 (4) 可知, ATD-SSI与MSDD的主要区别 在于 $h_{e}$ 是根据实际的目标强散射点密度自适应变化的, 不 需要事先知道散射点密度的先验信息, 提高了检测器对未 知散射点先验信息目标检测的鲁棒性。

\section{2. 强散射点个数估计}

令 $u_{k}=-2(N-1) \ln \left(1-w_{k}\right)$, 则在 $H_{0}$ 假设下, $u_{k}$ 的概 率密度函数为 ${ }^{[9]}$

$$
f_{u}(u)= \begin{cases}\frac{1}{2} \mathrm{e}^{-u / 2} & u \geq 0, \\ 0 & \text { 其它 }\end{cases}
$$

即可以认为 $u_{k}$ 是服从均值为 2 的指数分布的随机变量, 因此, $u_{k}$ 累积分布函数为

$$
F_{u}(u)=\left\{\begin{array}{lc}
1-\mathrm{e}^{-u / 2} & u \geq 0, \\
0 & \text { 其它 }
\end{array}\right.
$$

令 $x_{k}=F_{u}\left(u_{k}\right)$, 则 $X$ 在区间 $[0,1]$ 上服从均匀分布。由 于 $g(w)=-2(N-1) \ln (1-w)$ 和 $F_{u}(u)$ 在其定义域区间上是 单调递增的函数, 而 $W_{(1)} \leqslant W_{(2)} \leqslant \cdots \leqslant W_{(n)}$, 因此, $X_{(1)}$ $\leqslant \cdot X_{(2)} \leqslant \cdots \leqslant \cdot X_{(f)}, \quad \mathrm{AD}$ 检验的统计量可以表示为

$$
A_{K}=-K-\frac{1}{K} \sum_{i=1}^{K}(2 i-1) \ln \left[x_{(i)}\left(1-x_{(K+1-i)}\right)\right]
$$

$A_{K}$ 表示 $\left\{x_{k}\right\}$ 与均匀分布的适应程度, $A_{K}$ 越小表示适应 度越好。当目标不存在时, $x_{k}$ 服从均匀分布, $A_{K}$ 具有较 小的值; 当有目标存在时, 由于强散射点的作用, $u_{k}$ 不再 服从均值为 2 的指数分布, $x_{k}$ 偏离均匀分布, 从而 $A_{K}$ 值 也较大。由于强散射点回波对应序值较大的 $X$, 因此考虑 $n(n=1, \cdots, K)$ 个最小的 $x$, 计算其 $\mathrm{AD}$ 检验的统计量

$$
A_{n}=-n-\frac{1}{n} \sum_{i=1}^{n}(2 i-1) \ln \left[x_{(i)}\left(1-x_{(n+1-i)}\right)\right]
$$

可以认为 $A_{n}(n=1, \cdots, K)$ 达到最小值时对应的 $n$ 为杂波的 个数, 剩余的为强散射点, 考虑强散射点个数的估计值 $h_{\mathrm{e}} \in\{1,2, \cdots, K\}$, 以及强散射点个数估计值略大于真实
值时MSDD具有更好的检测性能 (这一点将在后面的仿真实 验中得到验证), 因此强散射点个数估计值可表示为

$$
h_{\mathrm{e}}=K-\arg \min _{n} A_{n}+1
$$

式中, $\arg \min _{n} A_{n}$ 表示取 $A_{n}$ 最小值对应的 $n$ 。

\section{4. 性能分析}

本节对MSDD和ATD-SSI进行了比较分析。仿真中, 采 用 $\Gamma$ 分布来表示混合分布 $f_{\tau}$

$$
f_{\tau}(\tau)=(L / b)^{L} \tau^{L-1} e^{-(L / b) \tau} / \Gamma(L), \tau \geq 0
$$

式中 $\Gamma(\cdot)$ 是Gamma函数, $b$ 是均值, $L$ 用来控制与正态统计 量偏离的程度。

$K$ 个距离单元内每个距离单元的目标平均功率为 $\sigma_{s}^{2}$, 杂波平均功率为 $\sigma_{\mathrm{c}}^{2}$ 。有目标散射点的距离单元 $\left(t \in \Theta_{h_{0}}\right)$ 回波用方差为 $\varepsilon_{t} \sigma_{s}^{2} K$ 的独立零均值复圆高斯随机变量建 模, 其中 $\varepsilon_{t}$ 表示距离单元 $t$ 的目标能量占目标总能量的比例。 本文考虑几种典型的MDS模型, $\varepsilon_{t}$ 的具体取值如表1所示。

表1 不同MDS模型的 $\boldsymbol{\varepsilon}_{t}$ 值。

\begin{tabular}{lllll}
\hline 目标距离单元 & 1 & 2 & $\cdots$ & $h_{0}$ \\
\hline Mode1.1 & $1 / h_{0}$ & $1 / h_{0}$ & $1 / h_{0}$ & $1 / h_{0}$ \\
Model. 12 & 0.5 & $0.5 /\left(h_{0}-1\right)$ & $0.5 /\left(h_{0}-1\right)$ & $0.5 /\left(h_{0}-1\right)$ \\
Mode 1.3 & 0.9 & $0.1 /\left(h_{0}-1\right)$ & $0.1 /\left(h_{0}-1\right)$ & $0.1 /\left(h_{0}-1\right)$ \\
Mode1.4 & 0.99 & $0.01 /\left(h_{0}-1\right)$ & $0.01 /\left(h_{0}-1\right)$ & $0.01 /\left(h_{0}-1\right)$ \\
\hline
\end{tabular}

当 $K, N, L$ 取不同值时, 文献 [10]对MSDD的检测性已 经进行了详细的分析, 因此, 本文主要比较分析对不同MDS 模型及散射点个数估计失配时对MSDD检测性能的影响, 以 及ATD-SSI的检测性能, 不失一般性, 假设 $K=15, N=2, L=1$, $P_{\mathrm{fa}}=10^{-4}$ 。信杂比定义为 $[10]$ :

$$
S C R=\left(\boldsymbol{p}^{\mathrm{H}} \boldsymbol{\Sigma}^{-1} \boldsymbol{p}\right) \sigma_{s}^{2} / \sigma_{c}^{2}
$$

当 $K=15, P_{\mathrm{fa}}=10^{-4}$ 时, 表 2 给出了 $h_{0}$ 取不同值时的MSDD 检测阈值。

表2 MSDD的检测阈值, $K=15, P_{\mathrm{fa}}=10^{-4}$ 。

\begin{tabular}{lllllllll}
\hline$h_{0}$ & 1 & 2 & 3 & 4 & 5 & 6 & 7 & 8 \\
\hline$T$ & 23.8 & 32.5 & 39.1 & 44.6 & 49.1 & 52.9 & 56.2 & 58.1 \\
$h_{0}$ & 9 & 10 & 11 & 12 & 13 & 14 & 15 & \\
$T$ & 61.2 & 63.1 & 64.6 & 65.8 & 66.7 & 67.3 & 67.6 & \\
\hline
\end{tabular}

分别采用表1中的4种MDS模型, 图1 (a) 图1 (d) 分析了 目标散射点个数估计值 $h_{\mathrm{e}}$ 对MSDD 检测性能的影响并与 ATD-SSI进行了比较, 其中, $h_{0}=3$ 。结果表明: 目标能量均 匀分布 (Mode1.1) 时, MSDD和ATD-SSI的检测性能最好, 目 标能量越集中, 检测性能越差。对于不同的目标模型, 当 
散射点个数估计值略大于真实值时 $\left(h_{\mathrm{e}}=4\right), \mathrm{MSDD}$ 具有最佳 的检测性能, 当散射点个数估计小于真实值时, MSDD信杂 比损失比较严重, 然而, 这一现象随着散射能量向单个距 离单元集中时得到改善。由图1(d) 可以看出, 即使 $99 \%$ 的 能量集中于一个单元时, 当散射点个数估计值小于实际值 时, 也会出现较大的信杂比损失。对于四种不同的目标模 型, ATD-SSI不需要事先知道目标散射点个数, 以及散射 点的强弱关系, 而其检测性能优于MSDD散射点个数估计匹 配时的检测性能。

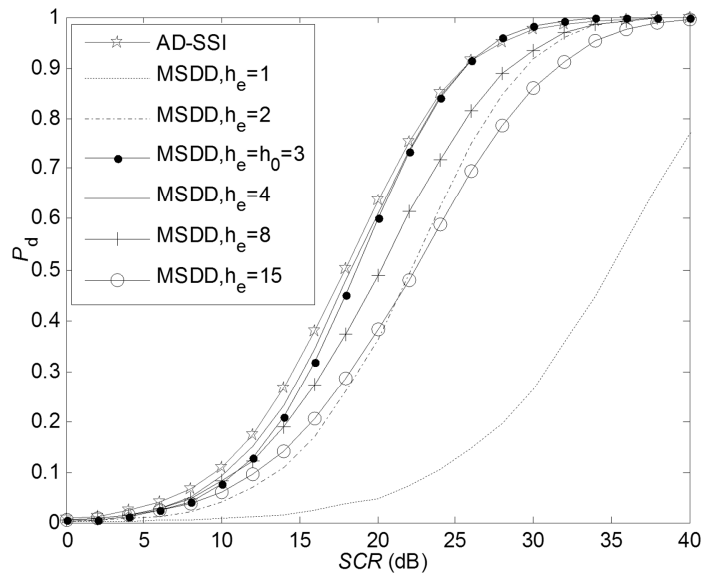

(a) Model. 1

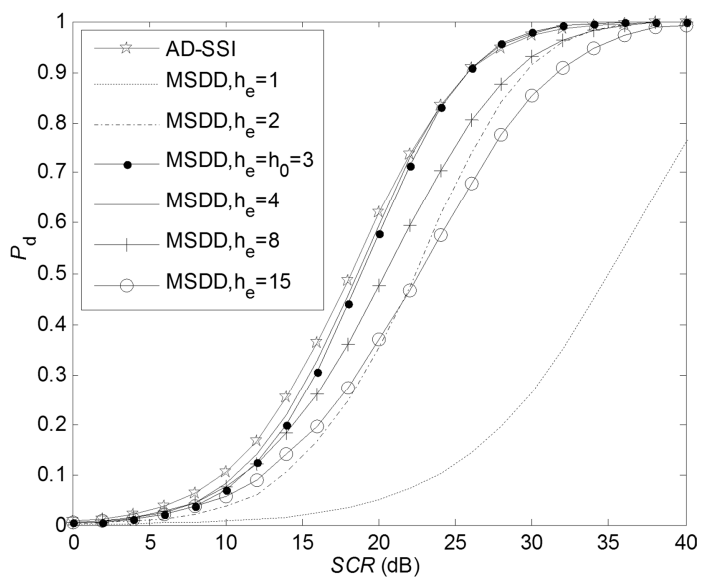

(b) Mode1. 2

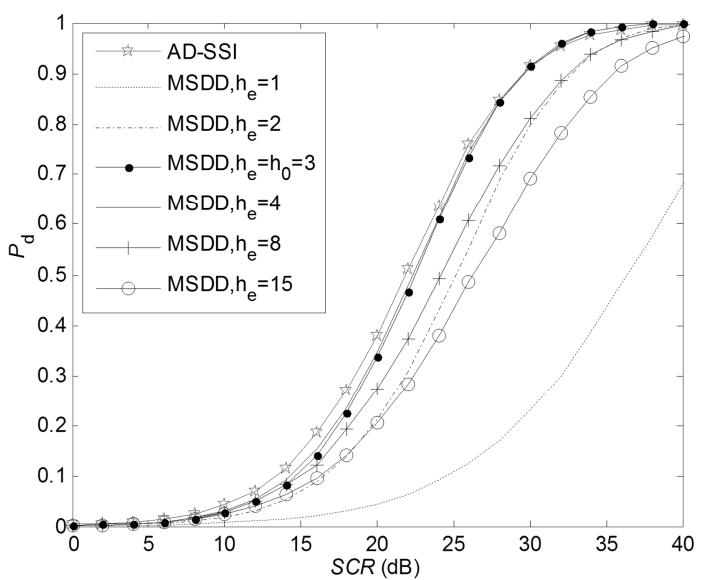

(c) Mode1. 3

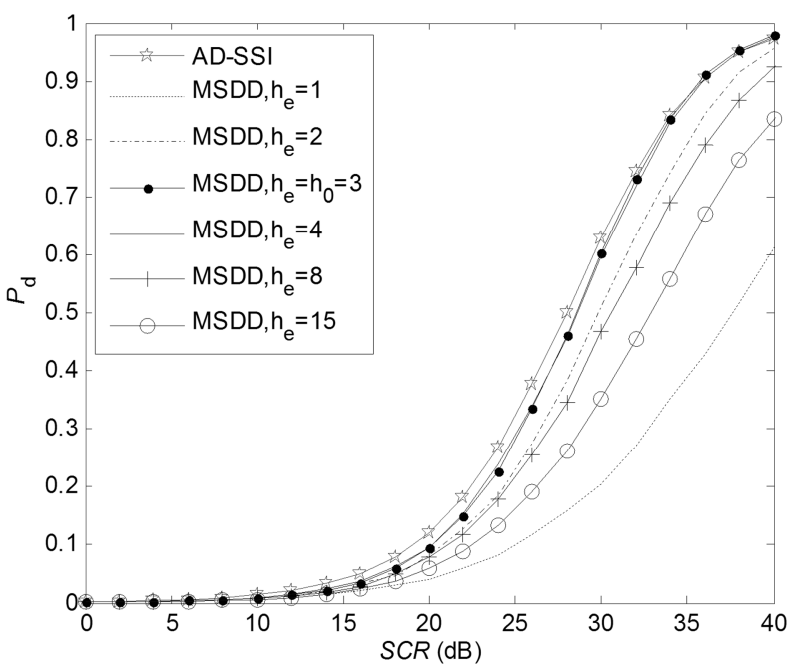

(d) Mode1. 4

图1 MSDD和ATD-SSI 与 $S C R$ 的关系曲线, $h_{0}=3$ 。

表3为某实测目标功率归一化一维距离像, 该目标有 4 个主散射点, 分布在不同的距离单元内, 且不同散射点的 强度不同, 针对该目标, 图2给出了MSDD检测概率与散射 点个数估计值的关系曲线, 并与ATD-SSI和NSDD检测性能 进行比较, 其中 $N=2, L=1, P_{\mathrm{fa}}=10^{-4}, S C R=20 \mathrm{~dB}$ 。从图2可 以看出, 当散射点个数估计值小于目标实际散射点个数时, MSDD存在严重的检测损失, 这与图1的结果是一致的; 当 散射点个数估计值略大于实际值时, MSDD的检测性能略优 于散射点个数估计匹配时的检测性能, 散射点个数估计值 继续增大时, MSDD的检测性能下降, 当 $h_{\mathrm{e}}=K$ 时, MSDD就等 价为NSDD。ATD-SSI能根据回波序列自适应的估计目标散 射点个数, 具有最佳的检测性能。

表3 某实测目标功率归一化一维距离像。

\begin{tabular}{lllllllll}
\hline 距离单元编号 & 1 & 2 & 3 & 4 & 5 & 6 & $\mathbf{7}$ & $\mathbf{8}$ \\
\hline 归一化功率 & 0 & 0.415 & 0 & 0 & 0.060 & 0 & 0 & 0 \\
距离单元编号 & 9 & 10 & 11 & 12 & 13 & 14 & 15 & \\
归一化功率 & 0 & 0 & 0.305 & 0 & 0.220 & 0 & 0 & \\
\hline
\end{tabular}

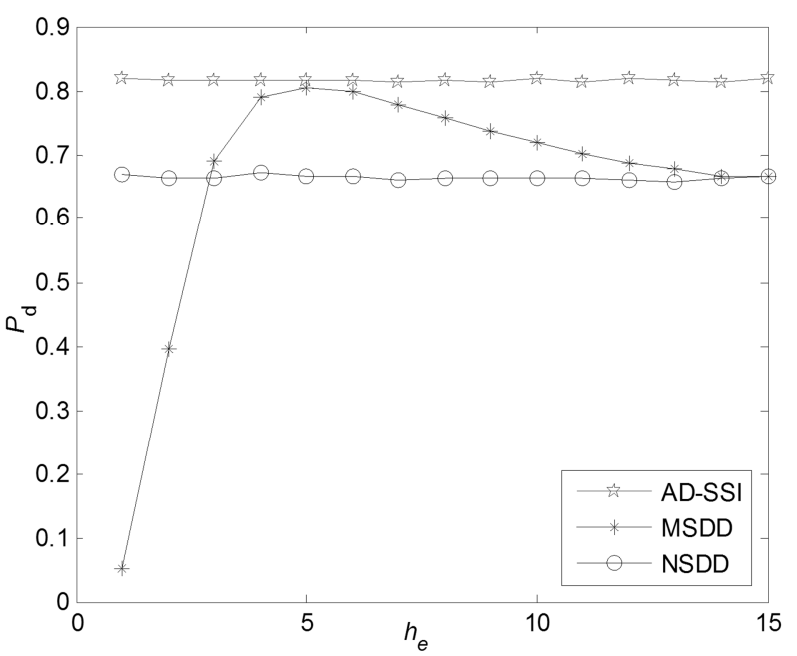

图2 $P_{\mathrm{d}}$ 与 $h_{e}$ 的关系曲线, $h_{0}=3, S C R=20$ 。 


\section{5. 结束语}

本文研究了SIRV非高斯杂波背景下的距离扩展目标 检测问题。针对MSDD检测器在估计目标散射点个数估计失 配时存在一定检测损失的问题，提出了一种能自适应估计 目标散射点个数的MSDD检测器（ATD-SSI）。针对不同的 MDS目标模型分析了MSDD在散射点估计匹配和失配时的性 能, 并和ATD-SSI比较, 结果表明MSDD得检测性能随着目 标能量向单个距离单元的集中而下降，并得出，即使 $99 \%$ 的能量集中于一个单元时, 当散射点个数估计值小于实际 值时, 也会出现较大的信杂比损失, 而ATD-SSI对于不同 的MDS目标模型都具有较好的检测性能。最后, 对MSDD的 失配性能进行了分析, 并与ATD-SSI和NSDD进行比较, 结 果表明, MSDD在散射点个数估计小于实际值时存在严重的 检测损失, 散射点个数估计大于实际值时的检测性能要优 于NSDD, 其中, 略大于实际值时能获得比估计匹配时更优 的检测性能, 而ATD-SSI能根据回波序列自适应的估计目 标散射点个数, 不需要已知目标散射点个数的先验信息, 具有较优的检测性能。

\section{参考文献}

[1] 顾新锋, 简涛, 何友, 等. 局部均匀背景中距离扩展目标的 GLRT 检 测 器 及性能分析 $[\mathrm{J}]$. 电子学 报, 2013, 41 (12) :2367-2373.

[2] 简涛, 何友, 苏峰, 等. 非高斯杂波下距离扩展目标检测器的 失配性能分析 [J]. 电子学报, 2010, 38 (7) : 1478-1482.

[3] Hughes P K. A high-resolution radar detection strategy [J]. IEEE Trans Aerospace Electron System, 1983, (19) : 663-667.
[4] Gerlach K, Steiner M J. Adaptive detection of range distributed targets [J]. IEEE Transactions on Signal Processing, 1999, 47(7): 1844-1851.

[5] Gerlach K, Steiner M, Lin F C. Detection of a spatially distributed target in white noise $[J]$. IEEE Signal Processing Letters, 1997, 4(7): 198-200.

[6] Conte E, De Maio A, Ricci G. GLRT-based adaptive detection algorithms for range-spread targets [J]. IEEE Transactions on Signal Processing, 2001, 49(7): 1336-1348.

[7] 戴奉周, 刘宏伟, 吴顺君. 一种基于顺序统计量的距离扩展 目标检测器 $[\mathrm{J}]$. 电子与信息学报，2009， 31 (10) :2488-2492.

[8] 顾新锋, 简涛, 何友, 等. 协方差矩阵结构的广义近似最大似 然估计 [J]. 应用科学学报, 2013, 31 (6) :585-592.

[9] Gerlach K. Spatially distributed target detection in non-Gaussian clutter [J]. IEEE Transactions on Aerospace and Electronic Systems, 1999, 35(3): 926-934.

[10] 简涛, 何友, 苏峰, 等. 非高斯杂波下修正的SDD距离扩展目 标检测器 $[J]$. 电子学报, 2009, 37 (12)：2662-2667. Jian Tao, He You, Su Feng, et al. Modified SDD-GLRT detector for range-spread targets in non-Gaussian clutter [J]. Acta Electronica Sinica. 2009, 37 (12) : 2662-2667. (in Chinese).

[11] Norouzi Y, Gini F, Nayebi M M. Non-coherent radar CFAR detection based on goodness-of-fit tests [J]. IET Radar Sonar Naving, 2007, 1(2) : 98-105. 